\title{
Invisibilidade da Pesquisa Clínica no Brasil: considerações a partir de fontes de informação em Ciência \& Tecnologia
}

\author{
Invisibility of Clinical Research in Brazil: Considerations on sources of \\ information in Science \& Technology
}

\section{Invisibilidad de la investigación clínica en Brasil: Consideraciones sobre fuentes de información en Ciencia y Tecnología}

Vinicius Pellizzaro Klein | klein.vinicius@gmail.com

Fundação Oswaldo Cruz (FIOCRUZ). Rio de Janeiro, Brasil.

\section{Resumo}

Apresenta a ampliação das atividades de pesquisa clínica no Brasil através do financiamento público recente. Correlaciona este fenômeno com o crescimento de informações científicas produzidas a respeito, bem como a demanda por acesso a estas informações de caráter público. Retrata a invisibilidade de informações como um obstáculo para a compreensão sobre o desenvolvimento atual da pesquisa clínica no país. Explora registros de fontes de informação em ciência e tecnologia sobre as atividades de pesquisa clínica de forma a evidenciar as atuais invisibilidades neste ambiente. Aponta, como um dos grandes fatores para a invisibilidade, a ausência de uma política de informação que, condicione uma cultura institucional de informar ostensivamente as atividades vinculadas. Considera o caráter ostensivo para além dos resultados gerados nas pesquisas, entendo que, o processo de construção destes resultados, também é uma informação de fundamental publicidade e acesso.

Palavras-chave: acesso à informação; fontes de dados; atividades científicas e tecnológicas; financiamento governamental; pesquisa médica translacional. 


\begin{abstract}
It presents the expansion of clinical research activities in Brazil through recent public funding. It correlates this phenomenon with the growth of scientific information produced about it, as well as the demand for access to this public information. It portrays the invisibility of information as an obstacle to understanding the current development of clinical research in the country. It explores records of sources of information in science and technology on clinical research activities in order to evidence the current invisibilities in this environment. It points out, as one of the great factors for invisibility, the absence of an information policy that conditions an institutional culture to ostensibly inform the related activities. Consider the ostensible character in addition to the results generated in the surveys, I understand that the process of building these results is also an information of fundamental publicity and access.
\end{abstract}

Keywords: access to information; data sources; scientific and technical activities; financing government; translational medical research.

\title{
Resumen
}

Presenta la ampliación de las actividades de investigación clínica en Brasil a través del financiamiento público reciente. Correlaciona este fenómeno con el crecimiento de informaciones científicas producidas al respecto, así como la demanda por acceso a estas informaciones de carácter público. En el caso de la investigación clínica en el país, se trata de un obstáculo para la comprensión del desarrollo actual de la investigación clínica en el país. Explora registros de fuentes de información en ciencia y tecnología sobre las actividades de investigación clínica para evidenciar las actuales invisibilidades en este ambiente. Señala, como uno de los grandes factores para la invisibilidad, la ausencia de una política de información que, condicione una cultura institucional de informar ostensiblemente las actividades vinculadas. Se considera el carácter ostensible más allá de los resultados generados en las encuestas, entiendo que el proceso de construcción de estos resultados, también es una información de fundamental publicidad y acceso.

Palabras clave: acceso a la información; fuentes de datos; actividades científicas y tecnológicas, financiación gubernamental; investigación em medicina translacional.

\section{Introdução}

A pesquisa Clínica pode ser definida como uma forma de investigação direcionada a um grupo de indivíduos voluntários através de terapias experimentais controladas junto ao acompanhamento e diagnóstico destes participantes ${ }^{1}$. O objetivo de avaliar empiricamente estes métodos terapêuticos em seres humanos é o de validar a segurança e eficácia de tais intervenções de forma que seja possível adotá-las como confiáveis para o tratamento curativo ou preventivo no cuidado à saúde. Dentre algumas terapias avaliadas nos estudos clínicos estão presentes dietas alimentares, treinos físicos, cirurgias, vacinas, fármacos, soros e aparelhos diagnósticos².

Tal pesquisa representa um elo importante entre postulados teóricos fornecidos pela pesquisa básica e a possível tradução destes conhecimentos em inovações materializadas na forma de serviços ou produtos ${ }^{3}$. Dada condição atende aos anseios globalizantes e mercantilistas da economia mundial de oferecer constantes soluções inovadoras ${ }^{4}$. Assim como também pode fundamentar a tomada de decisões de sistemas públicos de saúde na aquisição de novas tecnologias mais baratas e eficientes5. Por estas razões, a relevância e investimento neste tipo de estudo vêm sendo crescente tanto no Brasil quanto nos países desenvolvidos, ainda que estes locais possuam realidades distintas de agendas de pesquisa ${ }^{6}$. 
No cenário brasileiro em especial, a pesquisa clínica tornou-se recentemente um ponto central na Política Nacional de Ciência, Tecnologia e Inovação em Saúde (PNCTIS)7. Dentre as principais estratégias para sua promoção está a de aproximar a indústria nacional e o setor produtivo das instituições de pesquisa, buscando melhorar a capacidade inventiva do País ${ }^{8}$. Não por acaso, de 2005 até 2014, foram investidos no Brasil, através de uma articulação intersetorial entre os Ministérios da Saúde (MS) e Ciência Tecnologia e Inovação (MCTI), mais de R 120 milhões na contratação de estudos clínicos9. A ampliação das atividades neste segmento (decorrentes do progressivo fomento), consequentemente elevou a produção de conhecimentos científicos e registros de informação a respeito.

A dinâmica de produção de conhecimento na pesquisa clínica assume características singulares se comparada a outras dinâmicas. Ela tem seu início a partir dos conhecimentos científicos fornecidos por etapas anteriores a sua execução que, juntas, compreendem um complexo processo, denominado ciclo de Pesquisa \& Desenvolvimento $(\mathrm{P} \& \mathrm{D})^{10}$. Estas etapas precedentes são respectivamente a pesquisa básica, seguida de testes in vitro e experimentos com modelos animais. Por conseguinte, a Pesquisa Clínica se subdivide em quatro etapas, denominadas fases do estudo. Cada fase possui métodos e objetivos específicos, visando ao final, na última fase, que a terapia investigada seja comercializada. A Figura 1 sintetiza o ciclo da P\&D envolvendo as principais etapas componentes na produção de vacinas ${ }^{11}$.

Figura 1. Fluxo de Pesquisa \& Desenvolvimento de uma vacina

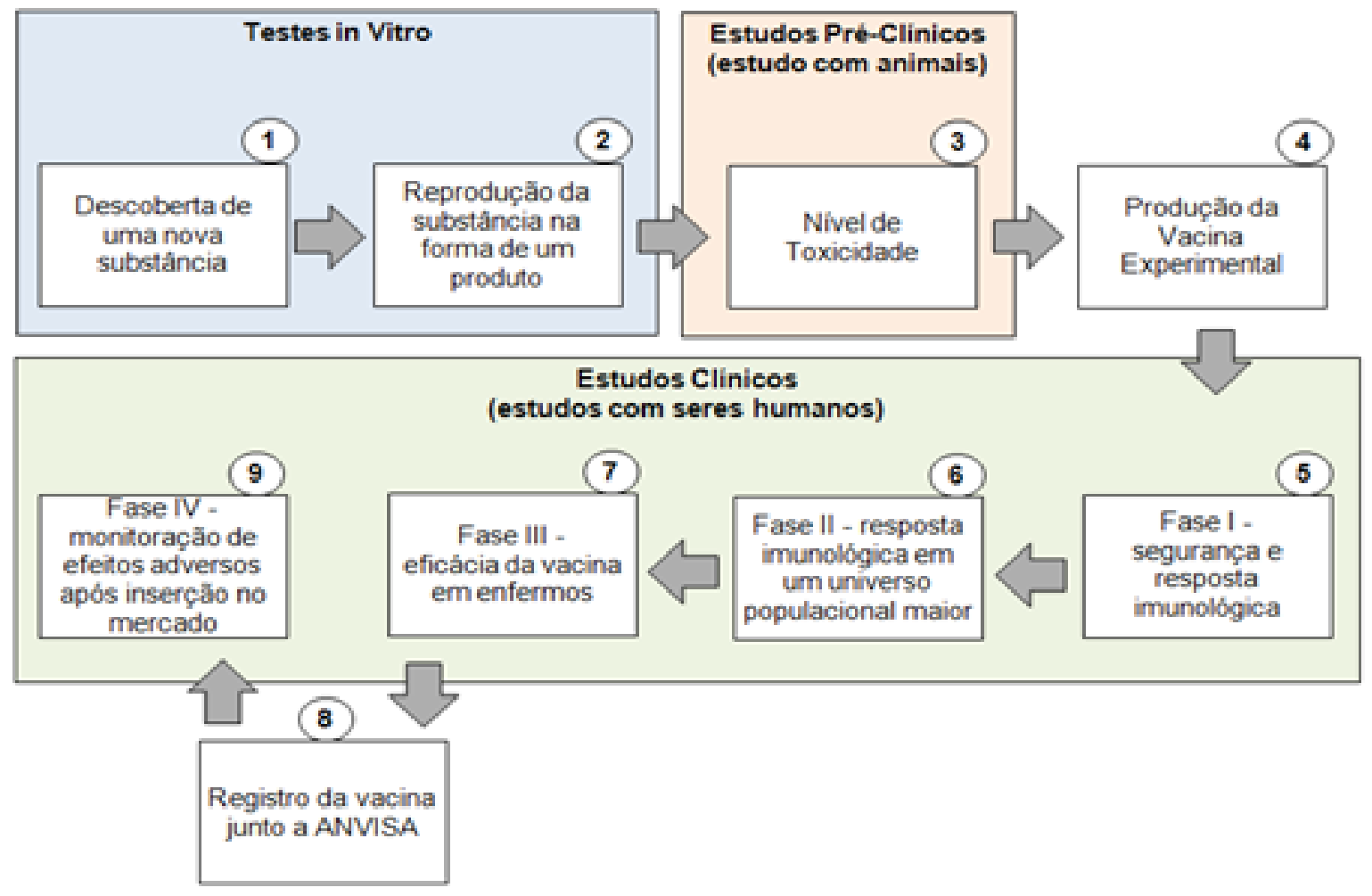

Fonte: elaborado pelo autor com base em Homma (2003)

No intuito de evitar a exposição desnecessária de indivíduos a estudos irrelevantes e garantir a integridade física destes voluntários participantes, há um detalhado controle na forma de relatar e documentar a formulação e condução dos estudos clínicos. O conjunto de dados sistematizados sobre cada pesquisa clínica permite a fiscalização e regulação pelos órgãos competentes das práticas efetuadas nas diferentes pesquisas desta natureza. Nas pesquisas realizadas em território nacional, os órgãos responsáveis por este controle são a Agência Nacional de Vigilância Sanitária (ANVISA) e o Comissão Nacional de Ética em Pesquisa (CONEP). A regulação da ANVISA é direcionada às instalações dos laboratórios, bem como aos materiais 
e/ou substâncias utilizadas no decorrer dos estudos clínicos. Já à CONEP cabe monitorar os projetos de pesquisa zelando pelos preceitos éticos que protejam o sujeito participante submetido ao estudo ${ }^{12}$.

O conjunto de exigências vigentes concerne, sobretudo, em documentar de forma transparente as intervenções por intermédio de dois instrumentos principais: o protocolo de pesquisa e o registro de ensaio clínico. O protocolo de pesquisa aborda o planejamento da investigação a ser desenvolvida e não é um instrumento de acesso ostensivo. Somente após aprovado pelas autoridades regulatórias competentes que, o ensaio clínico é conduzido e simultaneamente registrado em uma plataforma pública. O Ensaio clínico, portanto, representa o conjunto de procedimentos de investigação que seguem as determinações de um protocolo de pesquisa ${ }^{13}$. Através da condução do ensaio que são obtidas as respostas, isto é, os resultados sobre os efeitos de determinada terapia aplicada aos sujeitos de pesquisa selecionados. Tanto o protocolo como o ensaio necessitam apresentar um número relevante de dados no intuito de informar com clareza o estudo em questão. Abaixo segue uma síntese dos principais dados fornecidos por cada um destes instrumentos (Tabela 1) $)^{15,16}$.

Tabela 1. Síntese dos principais dados presentes protocolos e registros de ensaios

\begin{tabular}{|c|c|}
\hline Protocolo de Pesquisa Clínica & Registro de Ensaio Clínico \\
\hline Título do projeto & Código de registro de identificação do estudo \\
\hline Contato do pesquisador responsável & Período de realização do estudo \\
\hline Contato do patrocinador do estudo & Tipo de estudo realizado \\
\hline Descrição das hipóteses a serem testadas & Título do estudo \\
\hline Antecedentes científicos & Informação do patrocinador do estudo \\
\hline Descrição detalhada do projeto proposto & Fonte de apoio financeiro \\
\hline Análise de riscos e benefícios estimados & Condições de saúde \\
\hline Critérios para encerramento da pesquisa & Intervenção terapêutica realizada \\
\hline Detalhamento da infraestrutura disponível & Data do recrutamento dos participantes \\
\hline Orçamento financeiro do estudo & Número de recrutados \\
\hline Declaração de tornar público os resultados & Critérios para seleção dos recrutados \\
\hline População a ser estudada (faixa etária, sexo) & Critérios para exclusão do estudo \\
\hline Plano de recrutamento dos participantes & Desenho do estudo \\
\hline Termo de consentimento de participação & Contato para questões públicas \\
\hline Currículo dos pesquisadores & Contato para questões científicas \\
\hline Termo de compromisso do pesquisador & Contato sobre os centros de pesquisa \\
\hline
\end{tabular}

Fonte: Elaborado pelo autor

Como observado na Tabela 1, ambos os instrumentos indicam um conjunto de dados elementares para uma compreensão básica sobre a representação de cada estudo. Embora estes elementos registrados atendam às exigências de regulação e fiscalização e, portanto, cumpram o seu propósito, ainda assim uma parte considerável da trajetória de investigação dos estudos clínicos não é contemplada pelos dados expressos. Tal condição estimula uma reflexão sobre importantes questões que poderiam ser informadas sobre as pesquisas clínicas realizadas. Se adotarmos uma perspectiva construtivista ao olharmos sobre este caso, ou 
seja, de que o conhecimento científico produzido neste modelo de pesquisa é essencialmente uma construção social ${ }^{17}$, então os dados apresentados na tabela 1 são parte de um processo mais amplo.

Seguindo esta concepção, o registro de um ensaio clínico sintetiza um conjunto de informações produzidas, negociadas e compartilhadas ao longo da investigação através de acordos, conflitos e cooperações entre os pesquisadores e demais atores envolvidos. Logo, os registros clínicos tendem a uniformizar as diferentes experiências entre os muitos atores e lugares conectados, desconsiderando desta forma importantes elementos do percurso de construção da pesquisa. Portanto, questões como o tipo de atuação de cada centro de pesquisa coparticipante, as formas de colaboração utilizadas pelos integrantes, as relações com o patrocinador do estudo, são alguns pontos que merecem ser problematizados a partir destas considerações.

Aparentemente esta poderia ser uma discussão despropositada, dissociada do cidadão que mantem sua vida longe de complexas atividades de laboratórios de pesquisa. Entretanto, tais procedimentos por mais complexos que o sejam trazem consigo um desdobramento para a vida social, primeiramente por definirem por exemplo qual remédio devemos tomar em detrimento de um outro qualquer. Ademais, como parte constituinte de nossa cidadania, o acesso às informações públicas é um direito basilar ${ }^{14}$, embora, quando alcançado, ainda o seja com muita resistência. Portanto, a realização de uma pesquisa de financiamento público, independente de qual seja sua metodologia, deveria disponibilizar o maior número de dados possíveis. Esta é inclusive uma prerrogativa que tem encontrado voz por um movimento crescente denominado de Ciência Aberta ${ }^{18}$.

Frente ao exposto, o modelo de representação de ensaios e protocolos não são satisfatórios quanto a transparência de um processo mais amplo de produção do conhecimento, mesmo porque não são produzidos para este fim. Contudo, outras fontes de informação científica podem revelar, de forma complementar, elementos importantes para a compreensão sobre desenvolvimento destes estudos em uma perspectiva mais integral. O objetivo deste artigo é verificar se algumas das principais fontes públicas de informação em C\&T podem contribuir neste sentido.

\section{Procedimentos Metodológicos}

Como ponto de partida o primeiro passo foi a delimitação dos estudos clínicos de financiamento público a serem observados. A busca por estes estudos foi efetuada na página institucional das duas principais agências de fomento federais do Brasil: O Conselho Nacional de Desenvolvimento Científico e Tecnológico (CNPq) e a Financiadora de Estudos e Projetos (Finep). Em ambas as agências foram consideradas ao realizar a busca todas as chamadas de financiamento de pesquisa que contemplassem estudos clínicos.

Em relação ao sítio da Finep, as chamadas foram localizadas na seção denominada "editais", acessando em seguida o subitem "chamadas encerradas". Foram consultadas manualmente cada uma das chamadas encerradas ${ }^{19}$. Em relação a página do CNPq o método seguido foi similar. $\mathrm{O}$ acesso foi através do tópico “chamadas públicas", escolhendo a opção “encerradas”"20.

Em todos os casos de chamadas públicas localizadas no sítio das agências, cada uma das chamadas veio acompanhada por dois documentos. O primeiro, o edital da chamada (contendo as regras de seleção e contratação de estudos clínicos) e, o segundo, a lista com os projetos e pesquisadores contemplados.

Com base nas informações, sobretudo da lista dos contratados em cada edital, foram coletados em uma planilha os dados sobre os pesquisadores, suas instituições e o nome dos seus projetos. Os nomes obtidos dos pesquisadores permitiram localizar, em algumas ocorrências, um número maior de informações através de seus respectivos currículos armazenados na Plataforma Lattes ${ }^{21}$. Na seção do Lattes denominada "projetos de pesquisa", em determinados currículos com preenchimento deste campo, foram extraídos novos dados, tais como data, descrição do projeto, integrantes e financiadores. 
Por fim, a quarta fonte de informação acionada na coleta de dados foi o Diretório dos Grupos de Pesquisa no Brasil (DGPB). Este diretório concentra grande parte dos grupos de pesquisadores atuantes no Brasil nas diferentes especialidades científicas. A busca no $\mathrm{DGPB}^{22}$ pelo nome dos pesquisadores possibilitou (quando cadastrados) encontrar o nome dos grupos de pesquisa vinculados, as instituições parceiras e pesquisadores colaboradores.

Todos os dados citados quando obtidos foram extraídos das fontes consultadas (CNPq, Finep, Lattes e DGPB) e armazenados em uma planilha única, que por sua vez condensou o levantamento realizado, agrupando desta forma as informações de cada projeto e estabelecendo a correlação com os respectivos temas, instituições e pesquisadores colaboradores. O método aplicado permitiu evidenciar a transparência no relato das atividades de pesquisa clínica nestas fontes de informação em C\&T, inclusive quantificando padrões de comportamento tanto de visibilidade como invisibilidade dos dados.

\section{Resultados e Discussão}

Sobre a contratação de projetos de estudos clínicos, as agências de fomento analisadas anunciaram o total de 11 chamadas públicas presentes entre os anos de 2005 a 2014. Totalizaram 140 projetos contratados entre 103 pesquisadores representantes destes projetos. Das 11 chamadas realizadas, 7 foram através do CNPq e outras 4 pela Finep. A Tabela 2 enumera a relação destes editais.

Tabela 2. Chamadas de pesquisa clínica realizadas por CNPq e Finep de 2005 a 2014

\begin{tabular}{l|l}
\hline Chamadas pelo CNPq & Chamadas pela Finep \\
\hline CNPq 24/2005 & Finep 4/2005 \\
\hline CNPq 21/2006 & Finep 2/2007 \\
\hline CNPq 35/2008 & Finep 2/2008 \\
\hline CNPq 52/2009 & Finep 1/2013 \\
\hline CNPq 57/2010 & \\
\hline CNPq 67/2010 & \\
\hline CNPq 15/2013 & \\
\hline
\end{tabular}

Fonte: Elaborado pelo autor com base em CNPq (2015) e Finep (2015)

Comparando os dados fornecidos pelos órgãos de fomento ao longo das chamadas realizadas foi identificada uma ausência de critérios e padrões quanto à divulgação sobre estes projetos financiados. A omissão de dados importantes sobre o financiamento de projetos ocorre em todas as chamadas analisadas. Isto significa que, em nenhum dos casos observados, há uma compreensão satisfatória sobre a relação de pesquisadores, instituições e temas de pesquisa que receberam investimento financeiro das agências públicas. No caso específico da chamada 24/o5 (realizada pelo CNPq), não há qualquer menção sobre o destino dos recursos disponibilizados, o que configura um processo de pouquíssima transparência.

Em relação aos pesquisadores selecionados por estas chamadas, nos casos que há a divulgação do nome, somente é informada a relação dos pesquisadores principais, isto é, dos responsáveis pela submissão dos projetos de pesquisa apreciados pelas agências. Isto significa que a informação dos demais participantes das pesquisas não é fornecida pelas agências de fomento. De igual maneira, as instituições apresentadas são somente aquelas em que o pesquisador representante do projeto é vinculado. Neste cenário também é impossível conhecer as colaborações entre demais instituições envolvidas nos projetos. 
O Diretório do Grupo de Pesquisas (DGPB) da Plataforma Lattes, a princípio seria uma fonte que poderia expressar melhor a associação entre instituições e pesquisadores envolvidos nos projetos. Entretanto, ao acessá-lo, novas evidências apontam para a invisibilidade de informações. 10, dos 103 pesquisadores com projetos contratados, não possuem qualquer registro no Diretório. Somente 38 pesquisadores deste total relatam participação em grupos de pesquisa associados a atividades de pesquisa clínica, o que não foi possível assegurar se tais atividades são referentes aos projetos contratados. Outro ponto levantado foi a respeito da liderança e coordenação dos pesquisadores em grupos de pesquisa. Se, nos projetos contratados, os nomes fornecidos dos pesquisadores selecionados tratavam-se dos responsáveis pelos trabalhos submetidos, presumia-se que, ao analisar as participações destes mesmos pesquisadores no DGPB, eles estariam representados como líderes dos grupos de pesquisa ali cadastrados. Entretanto, em apenas 37\% dos casos estes pesquisadores figuram como líderes em grupos do DGPB.

No que concerne quanto ao assunto dos projetos contratados, o único dado (quando apresentado) se refere ao título dos projetos. Este importante dado somente é divulgado no edital Finep 1/13. Na Plataforma Lattes foi possível ter a ciência do nome de alguns projetos de pesquisa contratados, mas raros foram os casos em que estes dados foram preenchidos naquele local. Esta afirmação representa, em números, que somente em $49 \%$ dos projetos contratados se tem o conhecimento, por intermédio das agências de fomento ou Lattes, sobre o título dos projetos. As limitações impedem inclusive a busca por demais dados complementares, tendo em vista que o nome de um projeto, ou o que ele propõe, é um elemento básico para iniciar uma busca em qualquer outra fonte de informação. As lacunas de dados neste sentido impedem ou limitam substancialmente uma "ponte" com outras fontes externas de informação científica que possam elucidar de forma complementar sobre as características dos estudos clínicos.

Ao que o levantamento analisado indica, a invisibilidade no caso da pesquisa clínica é um problema sistêmico, cultural e supra institucional. Está presente nas agências de fomento, como também é manifesta, por parte dos pesquisadores, na utilização do Lattes e diretório de grupos ao preencherem seus perfis. A ausência de uma política de informação específica que instrua, oriente e imponha a publicidade dos dados, não pode ser desconsiderada. Se no caso dos protocolos e ensaios clínicos há uma determinação legal que obriga a uma sistemática de registro das atividades em pesquisa clínica, nos demais casos analisados neste trabalho, esta incumbência é algo facultativo.

A abertura opcional para o preenchimento descritivo de atividades de pesquisa em locais como o Lattes e o DGPB permite diversas omissões e divergências de informações por parte dos seus usuários. Esta inclusive é uma questão que nos convida a pensar enquanto pesquisadores das mais diversas competências sobre os dados abertos na Ciência. Como temos contribuído para dados mais democráticos na nossa produção científica? Ao realizarmos nossas pesquisas temos facultado pleno acesso ao que produzimos? Ou o anseio por dados abertos é algo que recai somente ao outro, ao estudo que queremos ter o acesso pormenorizado?

\section{Considerações Finais}

Por fim, algumas considerações podem contribuir para o enfrentamento do cenário indicado. A contratação estatal de projetos de pesquisa mediante o preenchimento adequado de fontes de informação nas principais plataformas e bases governamentais pode ser uma estratégia contra a invisibilidade. Uma maior participação de profissionais tais como bibliotecários, arquivistas e cientistas da computação e informação, ao atuarem multidisciplinarmente em grupos de pesquisa clínica, também representaria um caminho benéfico. Contudo, a mudança mais poderosa está em ampliar a noção de transparência, entendendo que esta significa não apenas a divulgação de resultados, mas a ampla informação de como os resultados são construídos. Isto representa reconhecer os contextos de produção e os coletivos acionados em cada empreendimento. Tornar este processo descritível e inteligível parece ser o maior desafio. Um desafio em que 
atualmente não há grandes limitações tecnológicas, mas sobretudo onde os obstáculos que se apresentam estão entorno da mobilização humana de promover meios mais democráticos e inclusivos.

\section{Referências}

1. Lousana G. Pesquisa clínica no Brasil. Rio de Janeiro: Editora Revinter; 2007.

2. Rosa EM., Lopes E.F. Pesquisa clínica: uma abordagem prática. São Paulo: Ícone Editora; 2011.

3. Guimarães R. Pesquisa translacional: uma interpretação. Ciênc. saúde coletiva [internet]. 2013 Jun [citado em 20 set. 2017];18(6):1731-1744. Disponível em: http://www.scielo.br/pdf/csc/v18n6/24.pdf

4. Petryna A. Experimentalidade: ciência, capital e poder no mundo dos ensaios clínicos. Horizontes Antropológicos. [internet]. 2011 Jan-Jun [citado em 20 set. 2017];17(35):127-160. Disponível em: http://www.scielo.br/pdf/ha/v17n35/v17n35a05.pdf

5. Paula AP, et al. Clinical investigations for SUS, the Brazilian public health system. Sao Paulo Med J. [internet]. 2012 Jan [citado em 20 set. 2017];130(35):179-186. Disponível em: http://www.scielo.br/ pdf/spmj/v130n3/v130n3a08.pdf

6. Brasil. Ministério da Saúde. Por que pesquisa em saúde? Brasília: Ministério da Saúde; 2007. Disponível em: http://bvsms.saude.gov.br/bvs/publicacoes/pesquisa saude.pdf

7. Brasil. Ministério da Saúde. Política Nacional de Ciência, Tecnologia e Inovação em Saúde. 2 ed. Brasília: Editora do Ministério da Saúde; 2008. Disponível em: http://bvsms.saude.gov.br/bvs/publicacoes/ Politica Portugues.pdf

8. Brasil. Ministério da Saúde. Agenda nacional de prioridades de pesquisa em saúde. 2 ed. Brasília: Editora do Ministério da Saúde; 2015. Disponível em: http://brasil.evipnet.org/wp-content/ uploads/2017/07/ANPPS.pdf

9. Klein VP. A pesquisa clínica no Brasil: uma análise preliminar a partir da RNPC. [dissertação]. Rio de Janeiro: Fundação Oswaldo Cruz; 2015. Disponível em: https://www.arca.fiocruz.br/handle/icict/14362

10. Oliveira, MA. Bermudez, JAZ. Osório-de-Castro, CGS. Assistência farmacêutica e acesso a medicamentos. Rio de Janeiro: Editora FIOCRUZ; 2007.

11. Homma A, et al. Desenvolvimento tecnológico: elo deficiente na inovação tecnológica de vacinas no Brasil. História, Ciências, Saúde - Manguinhos. [internet]. 2003 [citado em 20 set. 2017];10(suplemento 2):671-696. Disponível em: http://www.scielo.br/pdf/hcsm/v10s2/a11v10s2.pdf

12. Lousana, G. Accetturi, C. Pesquisa clínica: fluxos regulatórios no Brasil. Rio de Janeiro: Editora Revinter; 2013.

13. Organização Pan-Americana da Saúde. Boas Práticas Clínicas: Documento das Américas. Conferência Pan-Americana para harmonização da regulamentação farmacêutica, IV. República Dominicana, 2005. Disponível em: https://portal.fiocruz.br/sites/portal.fiocruz.br/files/documentos/documento das americas.pdf

14. Brasil. Constituição (1988). Constituição da República Federativa do Brasil. Brasília, DF: Senado Federal; 1988.

15. Comissão Nacional de Ética em Pesquisa. (Brasil). Resolução nº 196. Versão 2012. Aprova as seguintes diretrizes e normas regulamentadoras de pesquisas envolvendo seres humanos. Disponível em: http:// conselho.saude.gov.br/web comissoes/conep/aquivos/resolucoes/23 out versao final 196 ENCEP2012.pdf

16. Registro Brasileiro de Ensaios Clínicos: ReBEC. [internet]. Ministério da Saúde (BR); 2017 - [citado em 2017 set 20]. Disponível em: http://www.ensaiosclinicos.gov.br/rg/recruiting/

17. Latour, B. Ciência em ação: como seguir cientistas e engenheiros sociedade afora. 2 ed. São Paulo: UNESP; 2011.

18. Pinheiro, LVR. Do acesso livre à ciência aberta: conceitos e implicações na comunicação científica. RECIIS - Rev. Eletron. de Comun. Inf. Inov. Saúde. [internet]. 2014 Jun [citado em 20 set. 2017];8(2):153-165. Disponível em: https://www.arca.fiocruz.br/bitstream/icict/17107/2/8.pdf

19. Finep [internet]. Chamadas públicas: Ministério de Ciência, Tecnologia, Inovações e Comunicações. (BR); 2015 - [citado em 2017 set 20]. Disponível em: http://www.finep.gov.br/chamadas-publicas/ chamadaspublicas 
20. CNPq [internet]. Chamadas públicas: Ministério de Ciência, Tecnologia, Inovações e Comunicações. (BR); 2015 - [citado em 2017 set 20]. Disponível em: http://cnpq.br/chamadas-publicas

21. Plataforma Lattes [internet]. Ministério de Ciência, Tecnologia, Inovações e Comunicações. (BR); 2017 [citado em 2017 set 20]. Disponível em: http://lattes.cnpq.br/

22. Diretório dos Grupos de Pesquisa no Brasil [internet]. Ministério de Ciência, Tecnologia, Inovações e Comunicações. (BR); 2017 - [citado em 2017 set 20]. Disponível em: http://lattes.cnpq.br/web/dgp 